\title{
El juego de aquellos que juegan; reflexión acerca de la importancia del juego y su repercusión
}

\section{Artículo de reflexión}

\section{Igor Raphael Martínez Rebolledo}

Universidad de los Andes, Mérida, Venezuela

martinezigor1@gmail.com

Recibido: 27 de agosto de 2018

Aprobado: 4 de febrero de 2019

Cómo citar este artículo: Martinez Rebolledo, Igor (2019). El juego de aquellos que juegan; reflexión acerca de la importancia del juego y su repercusión en la participación social. Calle 14: revista de investigación en el campo del arte 14(26). pp.412-422. DOI: https:// doi.org/10.14483/21450706.15013

Agradecimiento e información sobre la naturaleza del manuscrito: El presente manuscrito es una reflexión producto del punto de partida del desarrollo de tesis doctoral en el Seminario de tesis del Doctorado en Ciencias de la Educación en la Universidad Pedagógica Experimental Libertador / Instituto de Mejoramiento Profesional del Magisterio, núcleo académico Mérida, Venezuela.

\section{(a) (1)}

https://creativecommons.org/licenses/by/4.0/deed.es 


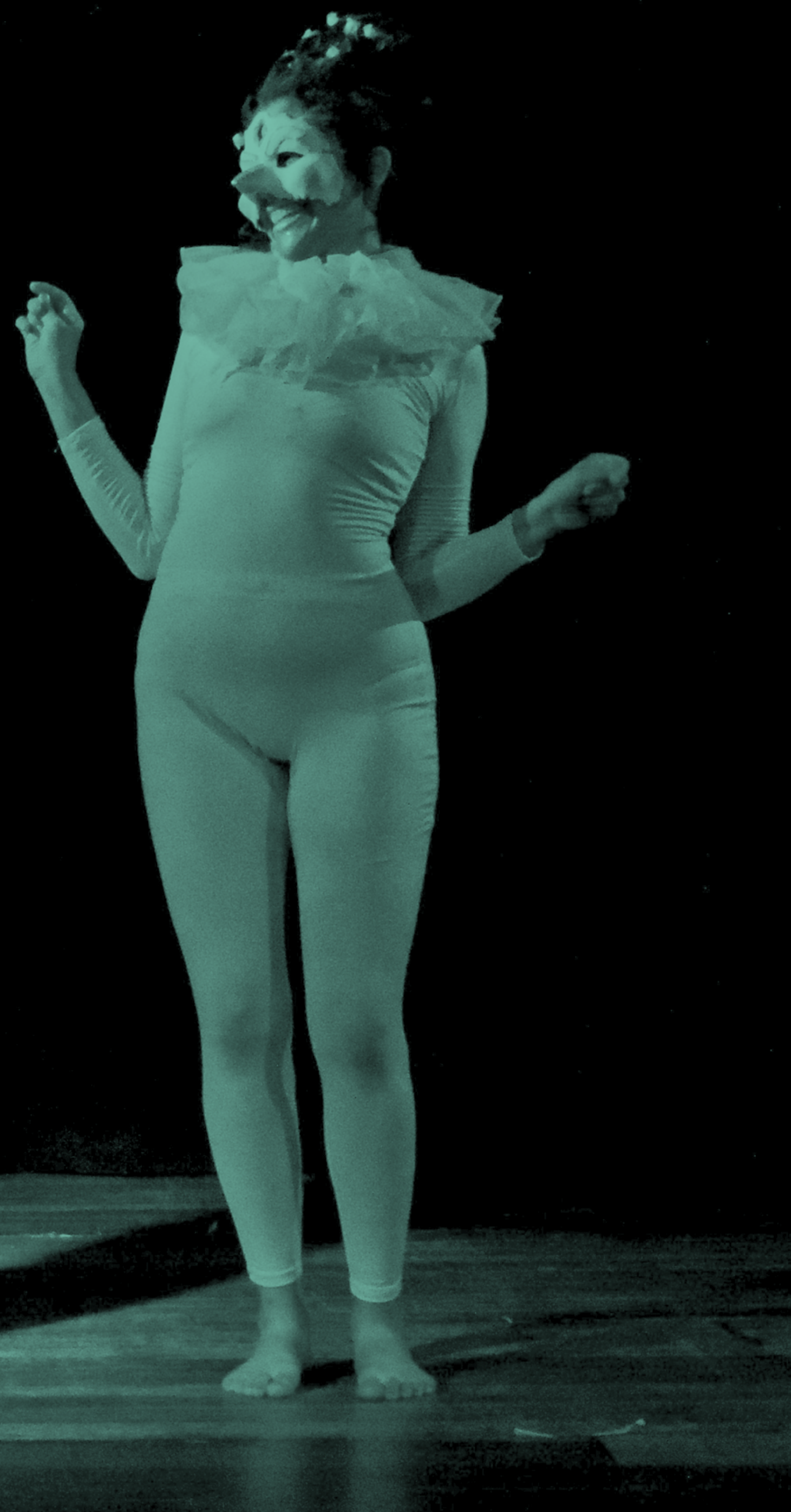


El juego de aquellos que juegan; reflexión acerca de la importancia del juego y su repercusión en la participación social

\begin{abstract}
Resumen
En las artes escénicas el juego resulta fundamental como técnica de entrenamiento, improvisación y punto de partida para el desarrollo del proceso creativo en la consecución de objetivos como la composición coreográfica e interpretación de un personaje para una puesta en escena. Es el juego también una herramienta social para entender el aprendizaje de un individuo y un camino para exteriorizar las necesidades de las personas; somos lo que aprendemos a través del juego. El presente artículo plantea una reflexión acerca de la importancia del juego como un elemento que define al individuo en la sociedad. Un espacio lúdico que desde nuestra infancia nos permite entender los diferentes roles que existen en la sociedad y los que ejerceremos en el futuro, así como también un instrumento que es fuente de expresión individual y colectiva que constituye un puente para generar la participación social.
\end{abstract}

\title{
Palabras clave
}

Juego; juego escénico; participación; participación social

The Game of Those Who Play: A Reflection on the Importance of Playing and its Impact on Social Participation

\begin{abstract}
In the performing arts, play is fundamental as a technique of training, improvisation and a starting point for the creative process when it wants to achieve objectives such as the choreographic composition and interpretation of a character for the stage. Playing is also a social tool to understand the learning of an individual and a path to externalize the needs of people; we are what we learn through playing. The present article proposes a reflection on the importance of play as an element that defines the individual in society: a playful space that from our childhood allows us to understand the different roles that exist in society and those that we will exercise in the future, as well as an instrument that is a source of individual and collective expression, building bridges to generate social participation.
\end{abstract}

\section{Keywords}

Playing; theater games; participation; social participation

Le jeu de ceux qui jouent : une réflexion sur l'importance du jeu et son impact sur la participation sociale

\begin{abstract}
Résumé
Dans les arts de la scène, le jeu est fondamental en tant que technique de formation, d'improvisation et point de départ du processus de création pour atteindre des objectifs tels que la composition chorégraphique et l'interprétation d'un personnage pour la scène. Le jeu est également un outil social pour comprendre l'apprentissage d'un individu et un moyen d'extérioriser les besoins des gens; nous sommes ce que nous apprenons en jouant. Le présent article propose une réflexion sur l'importance du jeu en tant qu'élément qui définit l'individu dans la société : un espace ludique qui permet dès notre enfance de comprendre les différents rôles qui existent dans la société et ceux que nous exercerons à l'avenir, aussi bien qu'un instrument source d'expression individuelle et collective, qui jette des ponts pour susciter la participation sociale.
\end{abstract}

\section{Mots clés}

Jeu ; jeu dramatique ; participation ; participation sociale 
O jogo dos que jogam: uma reflexão sobre a importância do jogo e seu impacto na participação social

\section{Resumo}

Nas artes cênicas, o jogo é fundamental como técnica de treinamento, improvisação e ponto de partida para o processo criativo no alcance de objetivos como a composição coreográfica e interpretação de um personagem para o palco. Jogar também é uma ferramenta social para entender a aprendizagem de um indivíduo e um caminho para exteriorizar as necessidades das pessoas; nós somos o que aprendemos jogando. 0 presente artigo propõe uma reflexão sobre a importância do jogo como elemento que define o indivíduo na sociedade: um espaço lúdico que desde a infância nos permite compreender os diferentes papéis que existem na sociedade e aqueles que exerceremos no futuro, assim como um instrumento que é fonte de expressão individual e coletiva, construindo pontes para gerar participação social.

\section{Palavras-chave}

Jogando; jogo dramático; participação; participação social

Pugllai paikuna pugllaikunamanda, allilla iuiarii chi pugllai imasami kauari socialpi

\section{Maillallachiska}

Suma ruraikuna pugllaikunapi chaianme tecnicasina entrenamientopi imasapas kallaringapa kauachingapa su runakuna kauagkunamanda chi pugllai kanme chasallata chi runa iachaikungapa i sug ñambe kauachii ministiskasina ningapa kanchi iachaikuikuna pugllaipe kai kilkaska kauachimi suma iuiarii imasami pugllaiua allilla karii tukuikunaua, imasami nukanchi uchulla kaura kauanchi i chasak kauangapa mas ñugpama u ñugpapa. Chasallata Rimangapa sapalla u achkakunape chasasina tukuikunaua andainakungapa.

\section{Rimangapa Ministidukuna}

Pugllai; pugllai kauachii; participación; participación socila 
Las artes escénicas a través de la historia, han tenido inmerso el asunto de el juego como el puente fundamental para entender, no solo el camino para desarrollar el aprendizaje, sino también el universo de expresiones a través de las cuales el ser humano exterioriza sus necesidades y se comunica con los demás. Es el juego dramático un puente de expresión que ofrece en su experiencia la oportunidad de que el ser humano pueda entenderse a sí mismo, relacionarse con los otros e incluso transformar la realidad según sean las necesidades humanas expuestas. Innes (1992) en su libro "El teatro sagrado. El ritual y la vanguardia" hace una analogía acerca de cómo se puede visualizar el juego escénico desde la mirada de Peter Brook (1925).

El concepto de teatro, según Brook, es como un "espacio vacío", cuya neutralidad imaginativa permite al actor desplazarse libremente por todo el mundo físico y entrar en una experiencia subjetiva para presentar "al hombre simultáneamente en todos sus aspectos" y hacer que el público participe colectivamente en una experiencia total... (Innes, 1992, p. 143)

Es el juego un espacio abierto al autorreconocimiento, un campo de aprendizaje en el que el individuo aprende de sí mismo, se reconoce y aprende de los demás. Y aunque en este caso se trata de el juego en la escena teatral, esta experiencia particular da cabida a que el público participe y se sienta a sí mismo como parte de la representación. Lo mismo sucede con el juego en el espacio natural humano, pues los individuos al emprender esta experiencia desde la infancia, proyectan y también contextualizan su realidad.

En el proceso enseñanza - aprendizaje, visto desde la mirada pedagógica de Mascaró (2001), se expresa que: "... el teatro es juego. Imitamos y aprendemos. Es difícil determinar dónde empieza el aprendizaje y donde termina el juego" (p. 9). Entendemos que en la infancia abrimos la puerta a la actividad lúdica y este proceso natural de divertimento nos conduce a reproducir, en nuestro universo infantil, acciones conocidas en donde el juego es un mundo particular establecido por uno mismo, donde se ejecutan roles que no nos son propios y que también conocemos e intuimos a partir de nuestro referente social y de la formación que poseemos.

Rivero (2016) en su artículo "El juego desde los jugadores" presenta una parte fundamental de la cultura hacia la creación de un nuevo sentido para comprender las acciones humanas, expresa lo siguiente:
(...) Es así como el juego ha conseguido un lugar en las ciencias de la educación en tanto actividad divertida que implica la participación de personas haciendo uso de habilidades que se pretenden estimular en las nuevas generaciones. De hecho, las propuestas educativas de juego, se suelen seleccionar primero las habilidades requeridas para participar y luego se define la forma del juego a proponer (mancha, escondida, carreritas, pelota, etc.). Aunque estas prácticas son habituales y resultan atractivas para los docentes, lo cierto es que desatienden la discusión respecto a qué se le llama estar jugando. (Rivero, 2016, p. 50).

Es el juego un punto de partida para abordar la representación de la realidad y es una de las herramientas por excelencia a través de las cuales un docente, principalmente de los primeros años de formación primaria, puede diagnosticar, a partir de acciones y situaciones simuladas, las características sociales del niño, sus posibilidades expresivas y de comunicación y su consideración acerca de su entorno más inmediato y del mundo que le rodea.

Mead (2008) en su obra "La filosofía del presente" nos habla de cómo el niño entiende su mundo social a través del juego. Explica en esta recopilación de manuscritos cómo el ser humano en su infancia va ocupando y comprendiendo roles a medida que representa y desarrolla su juego. Nos plantea que el niño va ejecutando los roles según su disposición, tomándolos de la sociedad adulta que le rodea y que en este proceso va comprendiendo su entorno y la sociedad a la que pertenece. Gana entendimiento y aprende a desarrollarse en el medio con absoluta comprensión de las situaciones que debe enfrentar al relacionarse con los demás.

(...) adoptar el papel del otro" es ver toda experiencia en un contexto nuevo, verla en función de lo que este contexto significa para, o proyecta sobre, los objetos - u objetivos - que su punto de vista define como centrales. (...) Es, precisamente, ese proceso de ajuste por el que un niño aprende a desempeñar los distintos papeles en una situación social, hasta que finalmente llega a juzgarse a sí mismo como persona responsable a la luz del valor que otros conceden a su conducta, y cuya autoridad su propia conciencia acepta ahora como tal al operar en nombre de ellos, lo que el ensayo final bosqueja (...). (Mead, 2008, p. 11) 


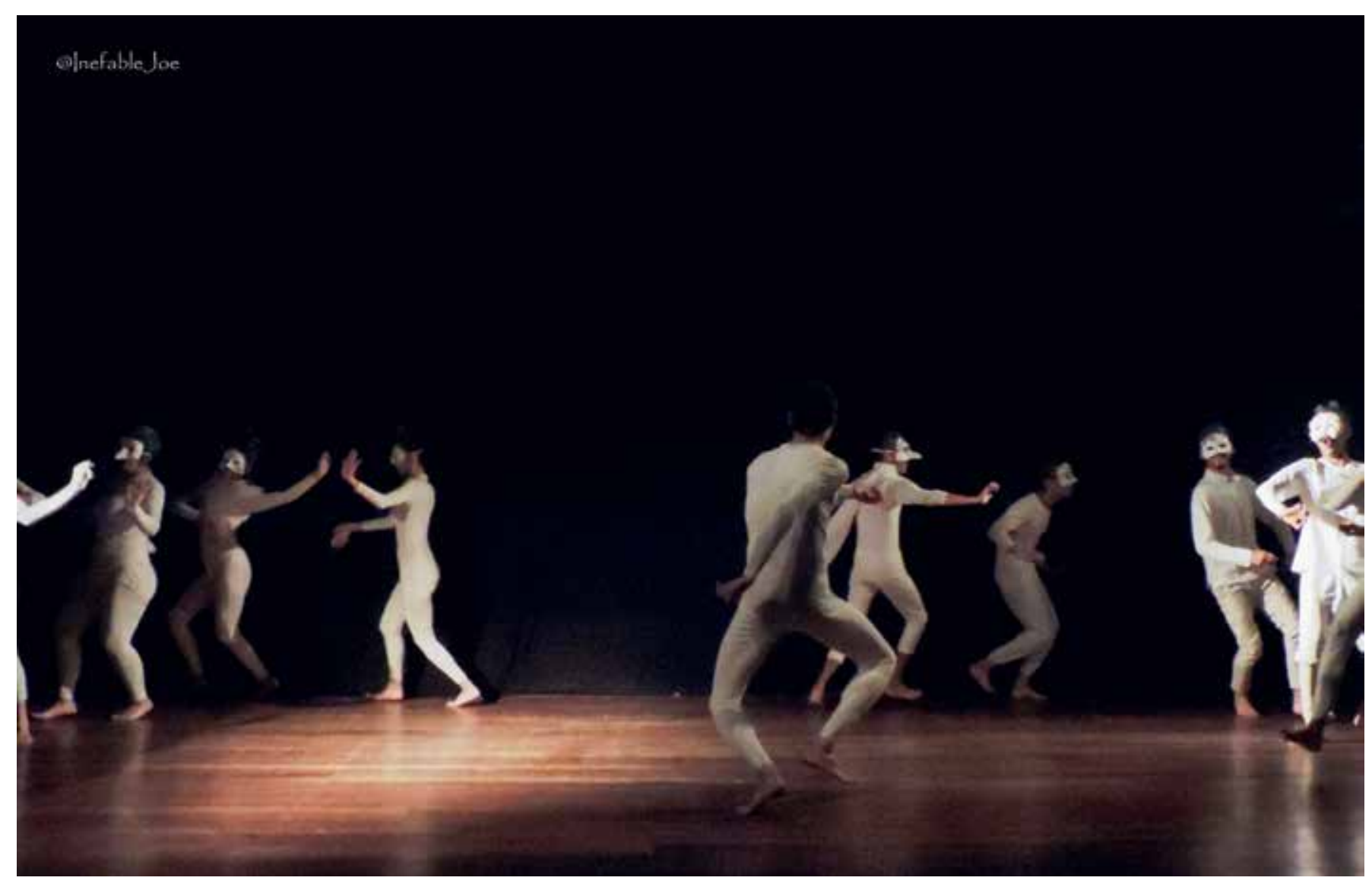

Imagen 1. Sin título. Joan Torres (2018).

La comprensión de la sociedad y su inherente conducta social viene determinada por "adoptar el papel del otro". La construcción de las interacciones entre los seres humanos van a depender del aporte que cada persona manifiesta de sí mismo, pero también del conocimiento que se tiene del otro, lo que le permitirá relacionarse, interactuar y hasta llegar a tener tolerancia y aceptación de lo que los demás piensan en relación con el mundo de las ideas que se comparten en el entorno social.

Esta visión de Mead (2008) hacia una fundamentación de las relaciones humanas en términos de "teoría de la comunicación", antecede e influencia a Habermas (2002) quien se orienta hacia el análisis de las condiciones de racionalidad humana en la acción social, haciendo énfasis en el sentido de comunicación y participación del ser humano a partir de la relación que establece con sus semejantes, basado en el lenguaje, que no tiene que ver directamente con el verbo escrito o hablado, sino en el que se expresa más allá a través del gesto, las señales y otros códigos de comunicación que le posibilitan entenderse y relacionarse con otros. Su crítica se orienta hacia la sociedad moderna por la intención de someter y debilitar la autonomía y racionalidad del individuo en pro de la determinación de una sociedad más integrada.

(...) La comunidad de seres morales que se dan a sí mismos sus leyes se refiere a todas las circunstancias que requieren regulación normativa con el lenguaje de los derechos y los deberes, pero sólo los miembros de esta comunidad pueden obligarse recíprocamente y esperar los unos de los otros comportamientos conformes a normas. Los animales se benefician de los deberes morales, que tenemos que respetar al tratar con criaturas que pueden sufrir por moral de ellas mismas. Con todo, no pertenecen al universo de los miembros que se dirigen mutuamente mandatos y prohibiciones reconocidos intersubjetivamente. Como deseo mostrar, la «dignidad humana» en estricto sentido moral y legal está ligada a esta simetría de las relaciones. (Habermas, 2002, p. 50)

Es la autonomía una pieza clave en la realización del ser humano y en su intención de generar un espacio de comunicación que constituye un medio apto para 


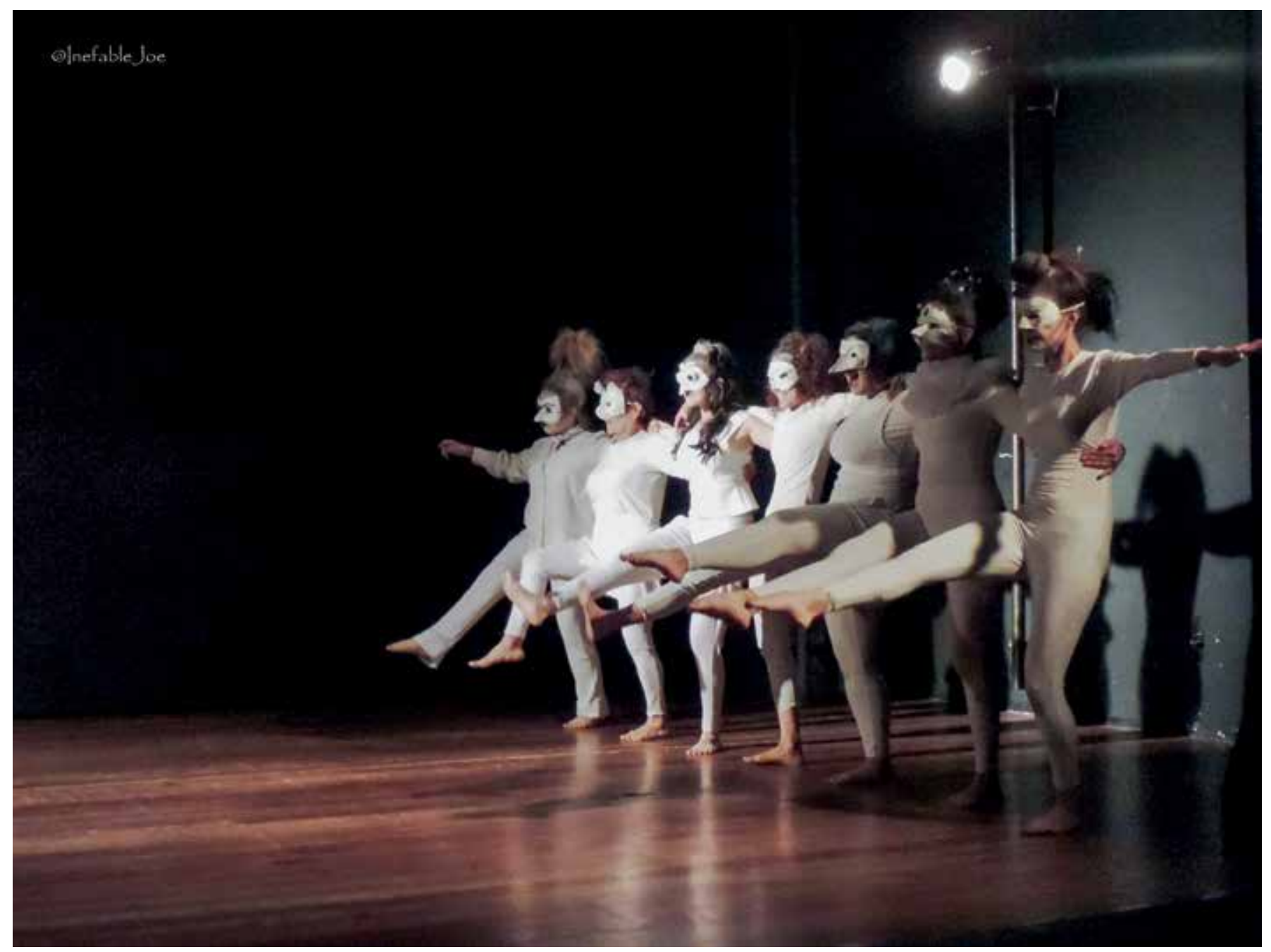

Imagen 2. Sin título. Joan Torres (2018).

la transmisión de ideas, se establece una relación de intercambio en el que se pueden transmitir mensajes que llegan a la conciencia en espacios comunes de convivencia y que encaminan los cambios que son necesarios para crecer y generar una vida más productiva. El modelo de racionalidad planteado por Habermas (2002), expone que una sociedad organizada debe entenderse como una colectividad en que las políticas públicas se acogen a través de un acuerdo común, de un diálogo en que las partes conciertan una serie de principios que se corresponden con los valores democráticos de una sociedad.

Estas políticas y valores, se orientan fundamentalmente hacia el proceso educativo, el cual debe conducirse hacia la emancipación de la humanidad, del progreso que va desde lo técnico al asunto moral. Dewey (1998) fiel defensor de la unión de la teoría y la práctica en el proceso formativo en pro de un enfoque progresista de la educación, le dio importancia al camino de traspasar lo meramente técnico hacia la personalización, la interpretación individual y la expresión de la proyección de la visión particular hacia lo general en el proceso formativo. La influencia de una vida presente, del aquí y el ahora, tan vital para el niño como la que vive en su hogar.

(...) Las escuelas son, en efecto, un método importante de la transmisión que forma las disposiciones de los seres inmaduros: pero son sólo un medio y, comparado con otros factores, un medio relativamente superficial. Sólo cuando hemos reconocido la necesidad de modos de tutela más fundamentales y persistentes podemos tener la seguridad de colocar los métodos escolares en su verdadero lugar. (...) Los hombres viven en una comunidad por virtud de las cosas que tienen en común; y la comunicación es el modo en que llegan a poseer cosas en común. Lo que han de poseer en común con el fin de formar una comunidad o sociedad son objetivos, creencias, 
aspiraciones, conocimientos -una inteligencia común- una semejanza mental como dicen los sociólogos. (Dewey, 1998, pp. 15-16)

Este vínculo que hace que los seres humanos se asocien desde la palabra común, comunidad y comunicación, ya que viven en convivencia a partir de los aspectos que les hacen comunes, es la cercanía con la acción social de Habermas (2002) y su sentido de participación y comunicación del ser humano y aunque la educación no ha sido un objeto importante de estudio en su obra, si resulta significativa la reflexión a partir de su interés por algunas cuestiones educativas presentes en el proceso de participación social entendida como la relación social del individuo a través de la cual expone los valores, el ejercicio y las condiciones culturales que tienen efecto en el sentido de integración, cooperación y emancipación, lo que les conduce a ser mejores en la sociedad en la que se desempeñan.

Como forma de organización ciudadana el individuo tiene la oportunidad de ser parte activa de acciones que contribuyen al trabajo en conjunto que resulta solidario y de integración en una comunidad, lo que les permite a las personas alcanzar propósitos de sustentabilidad, preservación y fortalecimiento de bases para alcanzar el progreso. Estas diversas formas de participación, según el ámbito en que se ponen en práctica, deben ser efectivas en su propósito y en las tres bases fundamentales para implementarla: el "poder"; que tiene que ver con la condición propicia para llevar adelante la acción comunitaria y la garantía de que las circunstancias están dadas para ponerla en práctica, el "querer"; orientado hacia la voluntad para alcanzar el propósito y el "saber"; el cual se enfoca hacia el conocimiento necesario para llevar adelante la actividad de participación social y ¿Por qué? y ¿Para qué? Se realiza.

Según el Manual metodológico de la Unesco, específicamente en lo concerniente a Indicadores Unesco de cultura para el desarrollo (2014); la participación social es igual a la "participación cultural" sobre la cual exponen lo siguiente:

Los beneficios personales que la participación cultural aporta a los individuos se pueden traducir en un fortalecimiento de la comunidad, ya que cuanto más elevados son los niveles de participación cultural en una sociedad determinada más se robustece su capital social. Como la participación cultural puede ayudar a los individuos a sentirse integrados en su comunidad, los riesgos de tensión social se reducen. Esto es especialmente importante en las sociedades multiétnicas y pluriculturales, en las que el acceso igualitario e inclusivo de todos sus grupos sociales a la vida cultural puede constituir un medio eficaz para fomentar el entendimiento mutuo. (p. 4)

La cultura desempeña sin duda un papel muy importante en el fortalecimiento de la participación en pro de una mejor calidad de vida y bienestar de los individuos y sus comunidades. Sin duda la participación social se encamina a partir de una serie de vectores que forman parte de la convivencia cultural del individuo, pues todas las necesidades que enfrenta un ciudadano en su comunidad tienen como arista de origen los valores culturales de la misma. Es así como la reinterpretación de valores, las aptitudes, convicciones a través de los cuales los individuos expresan su conducta social son fundamentales en el sentido de integración, afinidad y trabajo en común como fuerza para alcanzar beneficios en una sociedad.

El buen establecimiento de la relación social en una comunidad depende esencialmente de los beneficios que se le otorgan al individuo en la convivencia social, he ahí la importancia significativa de los valores humanos inmersos en la cultura de la participación. Pero volviendo al juego y a sus posibilidades en este tránsito de las relaciones sociales y de la participación social, es importante detenernos en lo que Marzán (1999) nos dice sobre el juego a partir de la mirada de Gadamer y su análisis acerca de este tema como intento por la recuperación de la mirada ontológica del ser humano. Marzán, expresa acerca de el juego que es: “(...) — carente de finalidad y repleto de posibilidades- que supera y envuelve a los sujetos que lo juegan" (p. 315). Y es que ciertamente, no hay un propósito por cuanto la experiencia del juego, independientemente de que existan pautas o premisas, posee en su desarrollo una serie de posibilidades expresivas y creativas que fácilmente pueden tergiversar el propósito inicial trayendo como consecuencia un resultado totalmente distinto a lo propuesto.

No es el fin de el juego lo importante de la experiencia, sino el proceso que se gesta en la innumerable cantidad de puertas que se van abriendo en su desarrollo. Los seres humanos, en nuestro actuar social, constantemente transitamos por el juego, aun inconscientemente en nuestra cotidianidad y cualquier circunstancia vivida, ya que cualquier acontecimiento puede ser transformado por nuestro pensamiento desde la elucubración de 
cómo debió haber sido para obtener un mejor resultado, se trata pues de un ejercicio imaginario, un acto lúdico de nuestra existencia, una autorrepresentación; y es esa la esencia de el juego en la participación social, de allí que Marzán (1999) lo presente de la siguiente manera:

La tentación que ofrece lo lúdico se encuentra en dejarse llevar por esa dinámica de la que no somos sus dueños, y en eso radica, precisamente, el elemento de riesgo y de incertidumbre que comporta. Sus reglas y el espacio delimitado en que se realiza nos instalan en un mundo propio y peculiar. Introducirse en él requiere que se acepte con solemnidad lo que nos plantea. Eso que nos encomienda ejerce una poderosa autoridad sobre nosotros, y en cuanto no se cumplen sus reglas o no se toman en serio, el juego se da por concluido (Marzán, 1999, p. 316).

Esta visión se complementa por lo planteado por Caillois (1986) en su libro: "Los Juegos y los hombres; las máscaras y el vértigo" en el que nos muestra una visión de el juego cuyo cometido es iluminarnos sobre esa parte del juego que angustia y conforta las manifestaciones fundamentales de la cultura, entiéndase todo aquello que representa el desarrollo del pensamiento, las artes, literatura e inspiración poética. De allí que se refiera a Huizinga (1933) autor del "Homo ludens"; obra a través de la cual, éste último, presenta una visión particularísima del juego, tan personal que resultó inédita hasta ese momento, pues se atreve a indagar por esta mirada particular del tema por primera vez, presentando una investigación sobre la exuberancia del ánimo del juego en el espacio de la cultura.

Resumiendo, podemos decir, por tanto, que el juego, en su aspecto formal, es una acción libre ejecutada "como si" y sentido como situada fuera de la vida corriente pero que a pesar de todo, puede absorber por completo al jugador, sin que haya en ella ningún interés material ni se obtenga en ella provecho alguno, que se ejecuta dentro de un determinado tiempo y de un determinado espacio (Huizinga. 2008, p. 53).

Esta especie de tentación que ofrece el juego lúdico a la idea de participar nos ha venido acompañando a través de la historia desde los tiempos en que Aristóteles (trad. en 2005) nos hablaba de la recepción de la obra como actor de participación del espectador. De allí deviene la denominada "catarsis"; término con el que daba explicación al acto de reflexión e identificación del ser humano una vez vivenciado como espectador el hecho dramático. La forma en que el drama influía en la existencia humana y de cómo gestaba en la humanidad la necesidad de redimir y depurar sus bajas pasiones en convivencia con otros espectadores a través de la reflexión desde el asunto ético, moral, social, religioso y cultural del drama y de cómo este era capaz de golpear y generar transformaciones en la vida del hombre de Grecia partiendo del sentido de la piedad y el temor.

Otra forma de participación a través del juego ocurrió con el teatro de Bertold Brecht para quien resultaba necesario que el espectador del drama se distanciara emocionalmente de la obra. Brecht, sostenía que el teatro debía mostrar las ideas de la realidad y que el público debía reflexionar ante este hecho en forma crítica y más objetiva, por lo que no debía existir una identificación o conexión emotiva entre el suceso dramático y el espectador a manera de no perjudicar el proceso reflexivo objetivo del acontecimiento del drama.

Ya en el siglo XX, se amplía el nivel de participación del espectador ante el juego dramático y es precisamente Gadamer quien introduce la idea de que "se juega al experimentar" y que el espectador participa a partir de la experiencia estética. Ejemplo de ello, como referencia experiencial del arte, es el acontecimiento que se vive con los "Penetrables" de Jesús Soto, artista plástico venezolano contemporáneo cuyas obras denominadas "Penetrables"; especie de rejilla metálica suspendida en el aire, de gran tamaño y de la que penden cientos de mangueras muy finas de caucho en color vivo, facilitan que el espectador traspase ese distanciamiento que suele existir entre la obra y el público permitiendo que este se integre a la obra misma, abriendo la puerta a las extraordinarias posibilidades de el juego.

En la experiencia el espectador forma parte de la obra, pues la misma se modifica una vez que este se incorpora a ella creando entre ambos una especie de complicidad, suerte de participación que desencadena un mundo de sensaciones en quien vivencia el hecho. Lo mismo que sucede hoy en el teatro, de allí la denominación de que "el teatro es juego", porque en la práctica del teatro se recrean situaciones para producir un espectáculo teatral o una improvisación para comprender y desarrollar la escena o crear el personaje. En este proceso en que el actor y el espectador se integran al mundo de la ficción de una representación teatral, se sumergen en un juego en el que se actúa en medio de juegos ilusorios; es justo el asunto que vivenciamos en nuestra infancia cuando jugamos como parte de nuestro desarrollo y aprendizaje, 


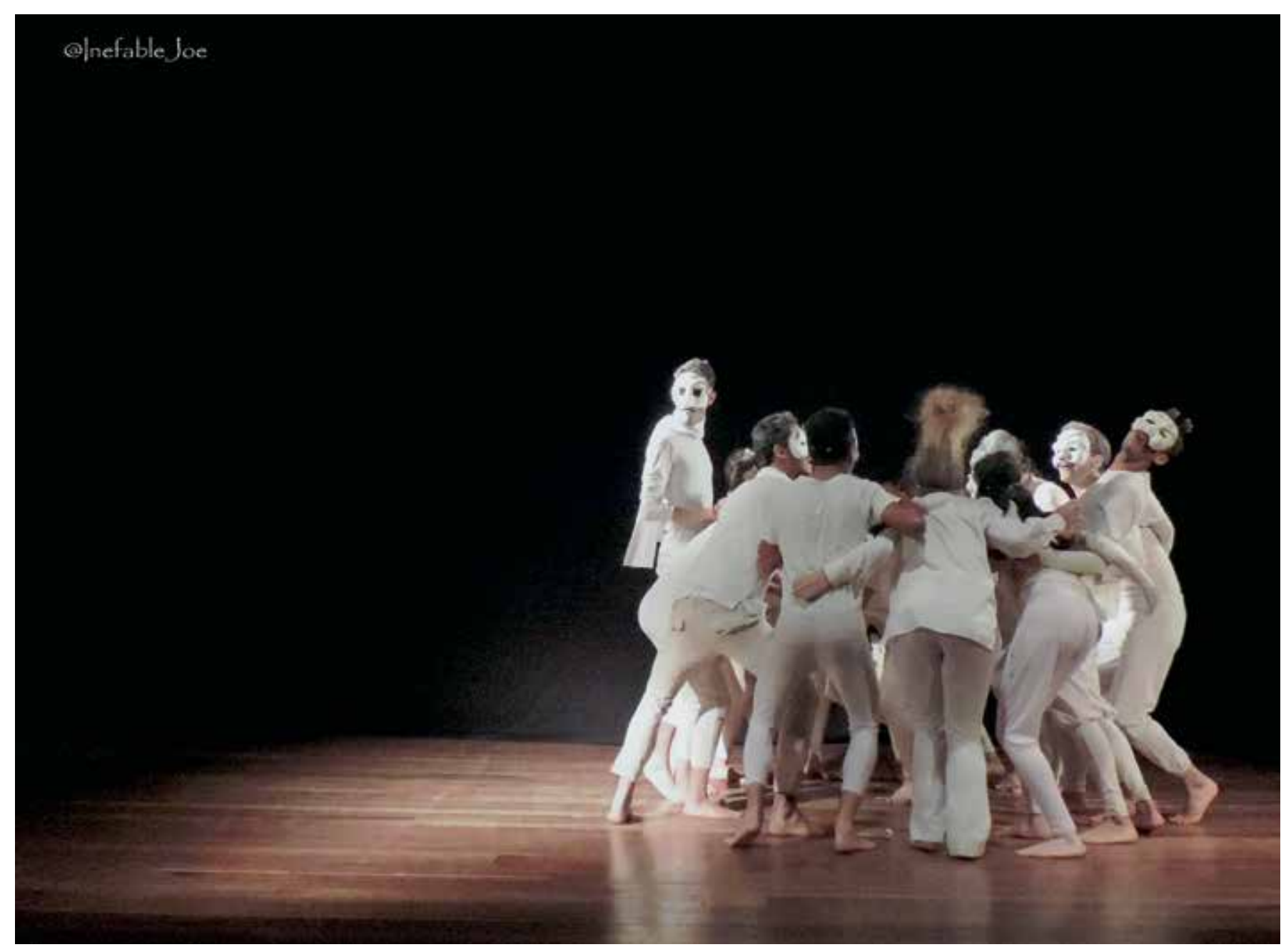

Imagen 3. Sin título. Joan Torres (2018).

solo que en los adultos el proceso se vive por necesidad de plantear otras situaciones. Lo cierto es que el juego nos permite dejar de ser nosotros mismos para convertirnos en otros personajes diferentes.

Es entonces el juego una forma de participación, una actividad inherente al desarrollo de la humanidad y su culturalidad, vinculado a la comunicación, belleza, arte, interpersonalidad, a lo sagrado, entre otros, pero con un impacto profundo en el ser de cada persona y en su cultura. Joan Huizinga nos señala en su libro "Homo Ludens" (2008) que el juego es el motor que desarrolla la cultura, ya que esta a su vez surge en modo de juego. Por lo que todo desarrollo cultural, en su inicio, está enmarcado por esta dinámica, pero que ese espacio lúdico se transforma y desarrolla en lo que se establecerá como legal, como parte del orden mismo del espacio cultural de la comunidad, es por ello que el juego nunca desaparece, sino que queda establecido en la actividad cultural como un valor propio. De allí deviene el impacto de el juego en la participación social.

\section{Referencias}

Aristóteles. (s.f /2005). La poética. Madrid, España: Alianza Editorial Madrid.

Barraza, A. (2003). Las ideas de Habermas y la educación. En Contexto educativo: revista digital de investigación y nuevas tecnologías. Universidad Pedagógica de Durango. DOI: https://www. researchgate.net/publication/28063505_Habermas_y_ la_Educacion [acceso 01 de marzo de 2018].

Caillois, R. (1986.) Los juegos y los hombres; la máscara y el vértigo. México: Fondo de cultura económica S.A. de C.V..

Gadamer, H. (1999). Verdad y método I. Salamanca, España: Ediciones sígueme.

Habermas, J. (2002). El futuro de la naturaleza humana. España: Ediciones Paidós Ibérica, S.A. 
(1981). Historia y crítica de la opinión

pública. Madrid: Editorial Gustavo Gili.

Huizinga, J. (2008). Homo Ludens. España: Editorial S.L. Fondo de Cultura Económica de España.

Innes, C. (1992). El teatro sagrado. El ritual y la vanguardia. México: Fondo de Cultura Económica, S.A. de C.V.

Lorca, O. (2005). Arte, juego y fiesta en Gadamer. En A Parte Rei: revista de filosofía, 41. ISSN 1137-8204, ISSN-e 2172-9069. [Consultado: 22 de junio de 2018] DOI: http://serbal.pntic.mec.es/ cmunoz11/lorca41.pdf

Marzán, C. (1999). Sobre el concepto del juego en Gadamer. Laguna: Revista de Filosofía, 6. ISSN
1132-8177. [Consultado: 22 de junio de 2018] DOI: http://publica.webs.ull.es/upload/REV\%20 LAGUNA/06\%20\%201999/20\%20(Carlos\%20

Marz\%C3\%A1n\%20Trujillo).pdf

Mascaró, P. (2001). Teatro el arte de la enseñanza. España: Editorial Ñaque.

Mead, G. (2008). La filosofía del presente. Madrid: Editorial Centro de Investigaciones sociológicas.

Ortega, R. (agosto, 2002) Lo mejor y lo peor de las redes de iguales: juego, conflicto y violencia. En Revista Interuniversitaria de Formación del Profesorado. [En línea] [Consultado 22 de junio de 2018] DOI: http:// www.redalyc.org/articulo.oa?id=27404406 